\title{
Novel Seleno- and Thio-Urea Containing Dihydropyrrol-2-One Analogues as Antibacterial Agents
}

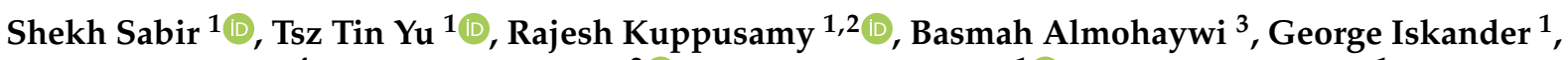 \\ Theerthankar Das ${ }^{4}$, Mark D. P. Willcox ${ }^{2}{ }^{\mathbb{D}}$, David StClair Black ${ }^{1}\left(\mathbb{D}\right.$ and Naresh Kumar ${ }^{1, *}$ \\ 1 School of Chemistry, The University of New South Wales, Sydney, NSW 2052, Australia; \\ s.sabir@student.unsw.edu.au (S.S.); tsztin.yu@unsw.edu.au (T.T.Y.); r.kuppusamy@unsw.edu.au (R.K.); \\ georgeiskanderb@gmail.com (G.I.); d.black@unsw.edu.au (D.S.B.) \\ 2 School of Optometry and Vision Science, The University of New South Wales, Sydney, NSW 2052, Australia; \\ m.willcox@unsw.edu.au \\ 3 Department of Pharmaceutical Chemistry, College of Pharmacy, King Khalid University, \\ Abha 61421, Saudi Arabia; bal-mohawe@kku.edu.sa \\ 4 Department of Infectious Diseases and Immunology, School of Medical Sciences, The University of Sydney, \\ Sydney, NSW 2006, Australia; das.ashishkumar@sydney.edu.au \\ * Correspondence: n.kumar@unsw.edu.au; Tel.: +61-29385-4698; Fax: +61-29385-6141
}

\section{check for} updates

Citation: Sabir, S.; Yu, T.T.; Kuppusamy, R.; Almohaywi, B.; Iskander, G.; Das, T.; Willcox, M.D.P.; Black, D.S.; Kumar, N. Novel Selenoand Thio-Urea Containing Dihydropyrrol-2-One Analogues as Antibacterial Agents. Antibiotics 2021, 10, 321. https://doi.org/10.3390/ antibiotics 10030321

Academic Editor:

Enrique Domínguez-Álvarez

Received: 1 March 2021

Accepted: 18 March 2021

Published: 19 March 2021

Publisher's Note: MDPI stays neutral with regard to jurisdictional claims in published maps and institutional affiliations.

Copyright: (c) 2021 by the authors. Licensee MDPI, Basel, Switzerland. This article is an open access article distributed under the terms and conditions of the Creative Commons Attribution (CC BY) license (https:// creativecommons.org/licenses/by/ $4.0 /)$.

\begin{abstract}
The quorum sensing (QS) system in multi-drug-resistant bacteria such as $P$. aeruginosa is primarily responsible for the development of antibiotic resistance and is considered an attractive target for antimicrobial drug discovery. In this study, we synthesised a series of novel selenourea and thiourea-containing dihydropyrrol-2-one (DHP) analogues as LasR antagonists. The selenium DHP derivatives displayed significantly better quorum-sensing inhibition (QSI) activities than the corresponding sulphur analogues. The most potent analogue $3 \mathbf{e}$ efficiently inhibited the las QS system by $81 \%$ at $125 \mu \mathrm{M}$ and $53 \%$ at $31 \mu \mathrm{M}$. Additionally, all the compounds were screened for their minimum inhibitory concentration (MIC) against the Gram-positive bacterium S. aureus, and interestingly, only the selenium analogues showed antibacterial activity, with $3 \mathbf{c}$ and $3 \mathbf{e}$ being the most potent with a MIC of $15.6 \mu \mathrm{M}$.
\end{abstract}

Keywords: antibiotic resistance; Pseudomonas aeruginosa; quorum-sensing inhibitors; dihydropyrrol2-one; organoselenium compounds; antibacterial activity

\section{Introduction}

Antimicrobial resistance (AMR) is a continuously rising problem and a major challenging threat to the health of the global population. The Centers for Disease Control and Prevention (CDC) and World Health Organisation (WHO) have estimated that by 2050, millions of people could die due to resistant microbial infections, and this could lead to another pandemic [1,2]. The antimicrobial activities of conventional antibiotics is achieved through the disruption of bacterial cell walls, or inhibition of bacterial nucleic acid, protein or folate synthesis [3,4]. Consequently, there is an increased selective pressure build-up in bacteria, which causes the development of antibiotic resistance through a variety of mechanisms, including the formation of biofilms, the elimination of antibiotics by efflux pumps, target gene mutations and reduced cell permeability [5-8]. Hence, there is a pressing need to discover new classes of antimicrobial therapeutics with novel modes of action [9].

Quorum sensing (QS) is a cell-density-dependent communication system between bacterial cells that facilitates their collective behaviour in response to changes that occur in their environment [10]. Quorum-sensing signalling molecules, also known as autoinducers (AIs), are produced by many bacterial cells. At high bacterial density, these small signalling molecules communicate and coordinate their collective behaviour by regulating several activities such as biofilm formation, the production of virulence factors (pyocyanin, rham- 
nolipids, and pyoverdine), swarming motility, and antibiotic resistance by modulating the transcription of several genes [11,12].

Several types of QS systems are present in different bacteria. For instance, Pseudomonas aeruginosa possesses three major interconnected QS systems, namely las, rhl and pqs, which are differentiated based on the chemical nature of the associated autoinducers. These signalling molecules activate their respective cognate protein receptors (LasR, RhR, and PqsR), which subsequently upregulates the activities of multiple genes responsible for virulence and biofilm formation. In addition, these QS systems can modulate the activities of each other; for example, the activities of $r h l$ and pqs systems are positively regulated by las [13]. The las system utilises an $N$-acyl-L-homoserine lactone (AHL) type of autoinducer, which binds and activates a LuxR-type transcriptional regulator receptor LasR. P. aeruginosa synthesises $\mathrm{N}$-(3-oxododecanoyl)-L-homoserine lactone (3OC12-HSL) as an AHL autoinducer, which binds to the LasI/LasR cognate receptor and modulates the expression of multiple genes responsible for the production of various virulence factors and biofilm formation. Therefore, LasR antagonists could be utilised as novel antivirulence agents for the treatment of infections caused by multi-drug-resistant bacteria such as P. aeruginosa [14,15].

Several natural and synthetic lactones, such as fimbrolides and halogenated furanones, have been reported as AHL mimics and QS inhibitors (Figure 1) [16-18]. These inhibitors can inhibit QS and biofilm formation. Structure-function analyses of previously reported furanone and dihydropyrrol-2-one (DHP)-based QSI inhibitors have revealed that the presence of a conjugated exocyclic double bond is critical for their inhibitory activity [19]. We have previously reported DHP-based AHL mimics that contain a five-membered lactam ring and an aryl (phenyl) group at the 4-position and an exocyclic double bond [20]. The stability of lactams from hydrolysis under normal physiological conditions makes them a better choice than the corresponding oxygen-containing (lactone) analogues. The DHP moiety is present in several classes of biologically important natural and synthetic molecules. In our recent work, we synthesised a series of thioether-containing DHP analogues as PqsR antagonists, and several of those analogues exhibited good pqs quorumsensing inhibitory (QSI) and biofilm inhibition activities against POA1 P. aeruginosa without affecting bacterial growth [21].

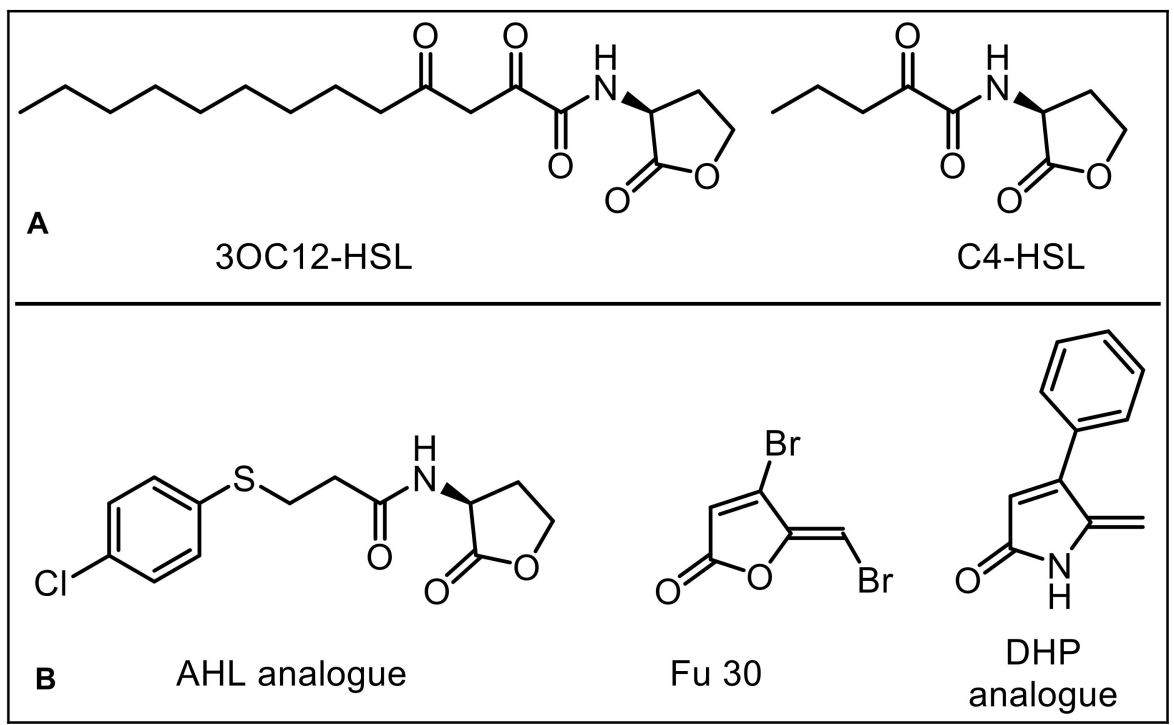

Figure 1. (A) AHL-based natural autoinducers of P. aeruginosa. (B) Synthetic AHL-based quorumsensing inhibitors (QSIs).

Organoselenium compounds have attracted considerable attention as biologically active compounds due to the low toxicity and antioxidant properties of selenium [22-24]. As an antioxidant, selenium acts as an essential element of several redox enzymes such as the enzyme glutathione peroxidase, required for catalysing several metabolic processes in 
the body [25]. Several organoselenium compounds have been reported as anticancer [26,27], antibacterial [28-31], and antiviral agents [32,33].

To elaborate on these earlier findings, the present investigation explored further modifications around the DHP motif via the introduction of selenourea and thiourea moieties attached directly to the C-5 exocyclic alkene of the pyrrol-2-one ring. The synthesised compounds were tested against the LasR QS reporter strain of P. aeruginosa (MH602). In addition, selenium-containing compounds were also tested for growth inhibition in the Gram-positive S. aureus (SA38) and the Gram-negative E. coli (K12). The screening results revealed that the selenium compounds were more active compared to the corresponding sulphur analogues.

\section{Results}

\subsection{Chemistry}

Synthesis of novel selenourea- and thiourea-containing DHP derivatives started with our recently reported key bromo DHP intermediates (2a-2i) [21]. In that previous work, it was reported that the bromo DHP compounds undergo an exchange reaction with different thiols and give thioether-containing DHP compounds under mild reaction conditions. In order to further explore the reactivity of the bromo DHP compounds, it was found that heating these bromo DHP compounds with selenourea at $60^{\circ} \mathrm{C}$ in acetone resulted in the corresponding DHP carbamimidoselenoate hydrobromide ( $\mathbf{3 a}-\mathbf{3 h}$ ) precipitating from the reaction mixture within $1-2 \mathrm{~h}$. The solid compounds could be easily isolated by simple filtration and subsequent washing with diethyl ether to give selenourea-containing analogues in $63-85 \%$ yields. Similarly, the corresponding DHP carbamimidothioate hyrobromide analogues $(\mathbf{4 a}-\mathbf{4 g})$ were synthesised in $48-96 \%$ yields by heating bromo DHP intermediates (2a-2i) with thiourea. However, in the case of thiourea, the reactions took longer (approximately 2-6 h) to complete (Scheme 1).

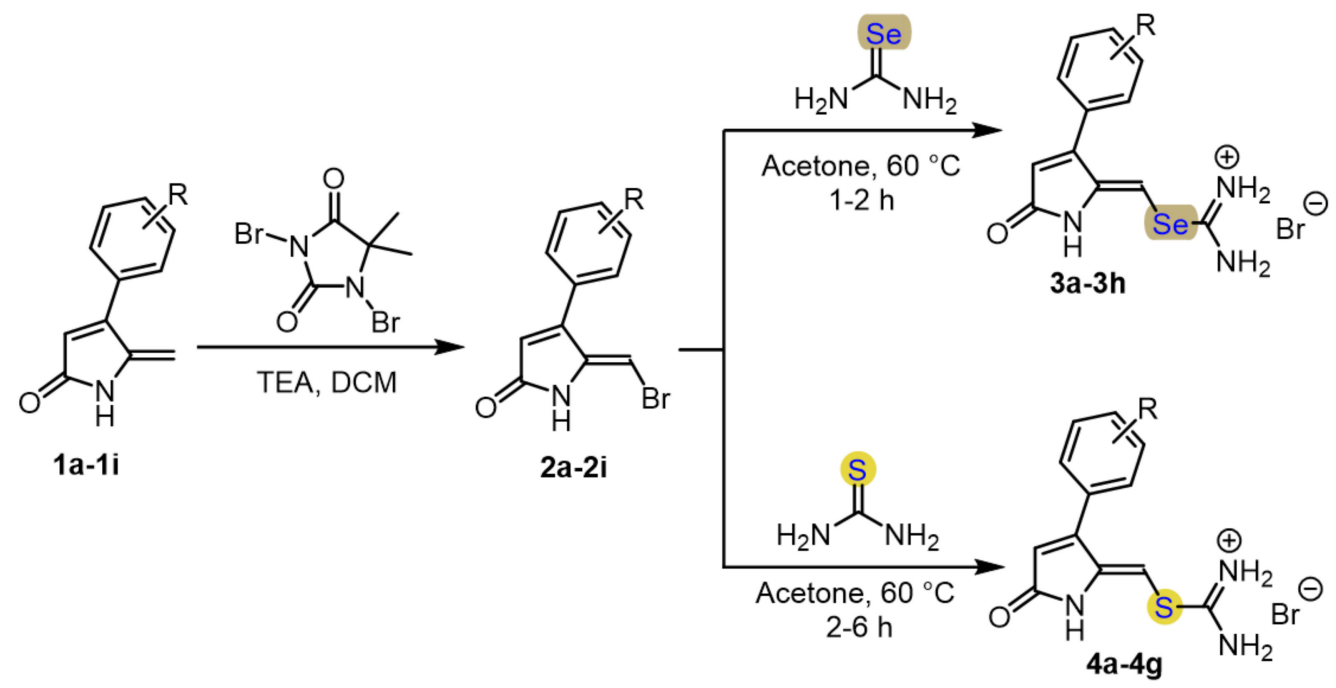

Scheme 1. General scheme for the synthesis of seleno-/thio-urea-containing dihydropyrrol-2-one derivatives.

The exchange reaction takes place readily with several different bromo DHP intermediates (2a-2i) and no significant effect of substitution on the aryl group of DHP was observed (Table 1). 
Table 1. Structures and yields of synthesised seleno-/thio-urea-containing dihydropyrrol-2-one compounds.

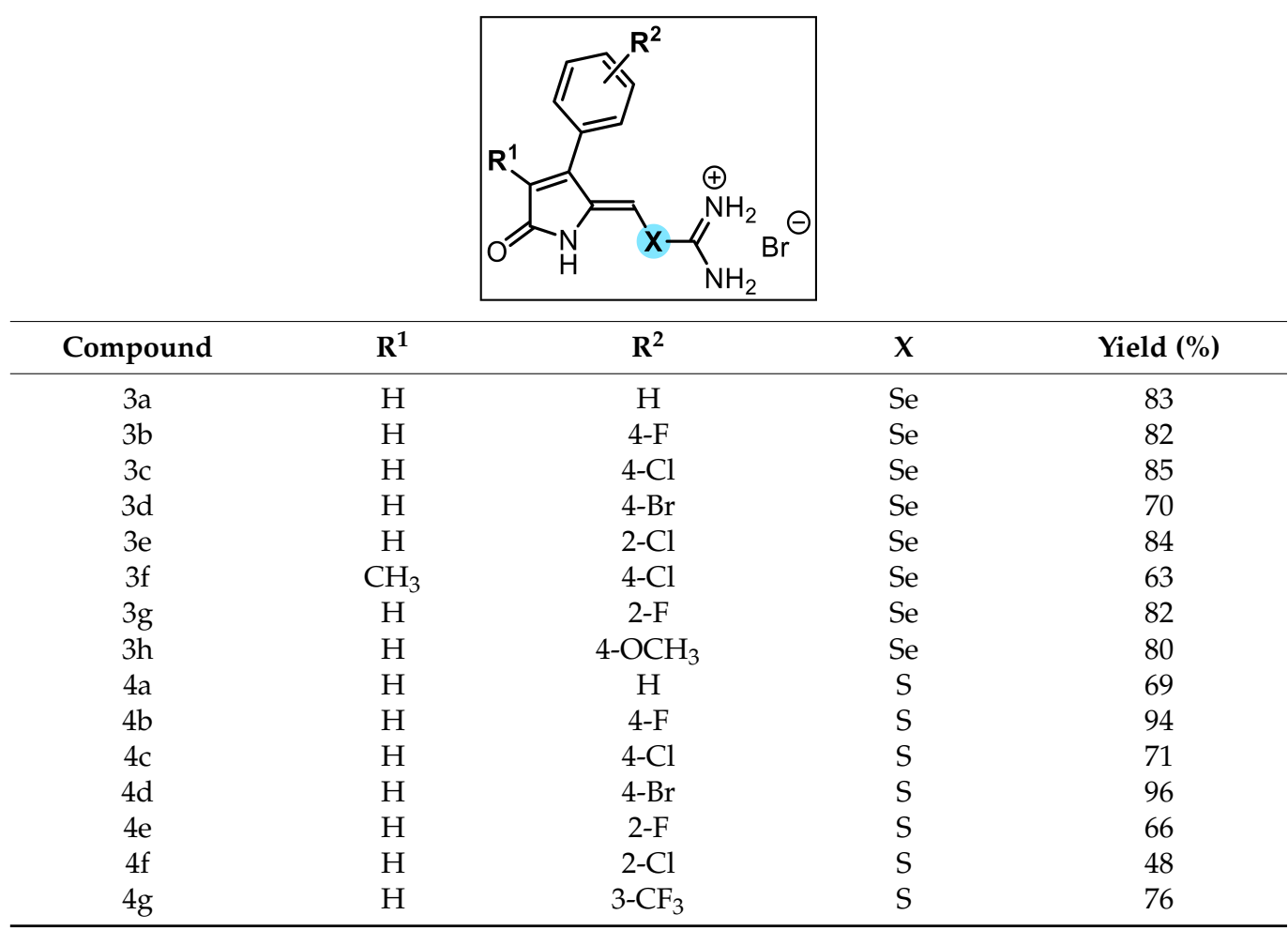

All the synthesised compounds were characterised by ${ }^{1} \mathrm{H}$ NMR, ${ }^{13} \mathrm{C}$ NMR, HRMS, and IR spectroscopy. To confirm the presence of a selenium atom in the structure, ${ }^{77} \mathrm{Se}$ NMR was carried out for compound $3 \mathbf{e}$ in DMSO- $d_{6}$, and this resulted in a single peak at $388.5 \mathrm{ppm}$, similar to the reported ${ }^{77} \mathrm{Se}$ NMR of other Se-containing compounds [34], which validates the presence of one selenium atom in the molecule (Supplementary Materials, File S12).

\subsection{Quorum-Sensing Inhibition Assay}

The novel selenourea and thiourea dihydropyrrol-2-ones were evaluated for their quorum-sensing inhibitory (QSI) activity using the P. aeruginosa MH602 $\mathrm{P}_{\text {las }} \mathrm{B}$ :gfp reporter strain, which harbours a chromosomal fusion of the lasB promoter to an unstable green fluorescent protein (GFP-ASV) reporter gene and responds to the AHL 3-oxo-dodecanoyl homoserine lactone (3oxo-C12-HSL) [35].

The GFP fluorescence level is a measure for the expression of AHL-mediated QS; therefore, the inhibition of the QS system by synthetic small molecules leads to the decrease in the fluorescence level of GFP, which can be directly correlated with the activity of those inhibitors [36].

The QSI activity of each compound was tested with three different micromolar concentrations $(125,63$, and $31 \mu \mathrm{M})$ by incubating with bacterial cultures at $37^{\circ} \mathrm{C}$ for $15 \mathrm{~h}$. The reduction in GFP fluorescence at $\lambda=535 \mathrm{~nm}$ was determined and expressed as a percentage of inhibition (QSI) compared to the negative control (only bacteria). A reported QS inhibitor Furanone 30 (Fu 30) was employed as a positive control.

The QSI activities of all the novel compounds were promising and concentration dependent, as shown in Table 2. Overall, the selenium-containing compounds (3a-h) displayed higher QSI activities than the corresponding sulphur analogues (4a-g). All the selenium-containing compounds showed greater than $60 \%$ QS inhibition at a $125 \mu \mathrm{M}$ concentration. The QSI activity of selenium compound 3a, which contained an unsubstituted phenyl group at the C-4 position, produced $80 \%$ inhibition at $125 \mu \mathrm{M}$ and $63 \%$ inhibition at $31 \mu \mathrm{M}$. The most potent compound was $3 \mathbf{e}$ with 2-Cl substitution at the phenyl ring, producing $81 \%$ inhibition at $125 \mu \mathrm{M}$ and $53 \%$ inhibition at $31 \mu \mathrm{M}$ (Figure 2). The $4-\mathrm{Cl}$ 
phenyl analogue $3 \mathrm{c}$ produced slightly lower activity of $74 \%$ at $125 \mu \mathrm{M}$ and $42 \%$ at $31 \mu \mathrm{M}$. The substitution at the C-3 position of lactam with a methyl group led to a decrease in activity as compound $3 \mathrm{f}$ showed only $69 \%$ inhibition at $125 \mu \mathrm{M}$. The selenium compound 3h (4-methoxy phenyl) exhibited QS inhibition of $80 \%$ at $125 \mu \mathrm{M}$ and $53 \%$ at $31 \mu \mathrm{M}$, and this suggests that there is no or a very small impact of different substitutions of the C-4 aryl group on the overall QS inhibition activity of these compounds.

Table 2. \% LasR QS inhibition at different concentrations of inhibitors 3a-3h, $\mathbf{4 a}-\mathbf{4 f}$, and reference fu 30.

\begin{tabular}{|c|c|c|c|}
\hline \multirow{2}{*}{ Compound } & \multicolumn{3}{|c|}{ P. aeruginosa MH602 } \\
\hline & $125 \mu \mathrm{M}$ & $61.5 \mu \mathrm{M}$ & $31 \mu \mathrm{M}$ \\
\hline $3 a$ & $80 \pm 4$ & $70 \pm 8$ & $63 \pm 3$ \\
\hline $3 b$ & $67 \pm 2$ & $43 \pm 6$ & $38 \pm 6$ \\
\hline $3 c$ & $74 \pm 2$ & $52 \pm 3$ & $42 \pm 4$ \\
\hline $3 d$ & $76 \pm 1$ & $58 \pm 6$ & $42 \pm 4$ \\
\hline $3 e$ & $81 \pm 5$ & $65 \pm 5$ & $53 \pm 2$ \\
\hline $3 f$ & $69 \pm 6$ & $49 \pm 6$ & $37 \pm 3$ \\
\hline $3 g$ & $66 \pm 7$ & $49 \pm 4$ & $38 \pm 4$ \\
\hline $3 \mathrm{~h}$ & $80 \pm 6$ & $64 \pm 7$ & $53 \pm 4$ \\
\hline $4 a$ & $50 \pm 1$ & $44 \pm 0$ & $37 \pm 1$ \\
\hline $4 b$ & $54 \pm 3$ & $38 \pm 13$ & $34 \pm 5$ \\
\hline $4 c$ & $70 \pm 3$ & $48 \pm 5$ & $35 \pm 3$ \\
\hline $4 d$ & $74 \pm 2$ & $54 \pm 5$ & $41 \pm 5$ \\
\hline $4 \mathrm{e}$ & $40 \pm 4$ & $29 \pm 7$ & $23 \pm 9$ \\
\hline $4 \mathrm{f}$ & $63 \pm 1$ & $55 \pm 6$ & $49 \pm 11$ \\
\hline $4 g$ & $56 \pm 2$ & $42 \pm 2$ & $34 \pm 3$ \\
\hline $\mathrm{Fu} 30$ & $96 \pm 2$ & $91 \pm 3$ & $93 \pm 2$ \\
\hline
\end{tabular}

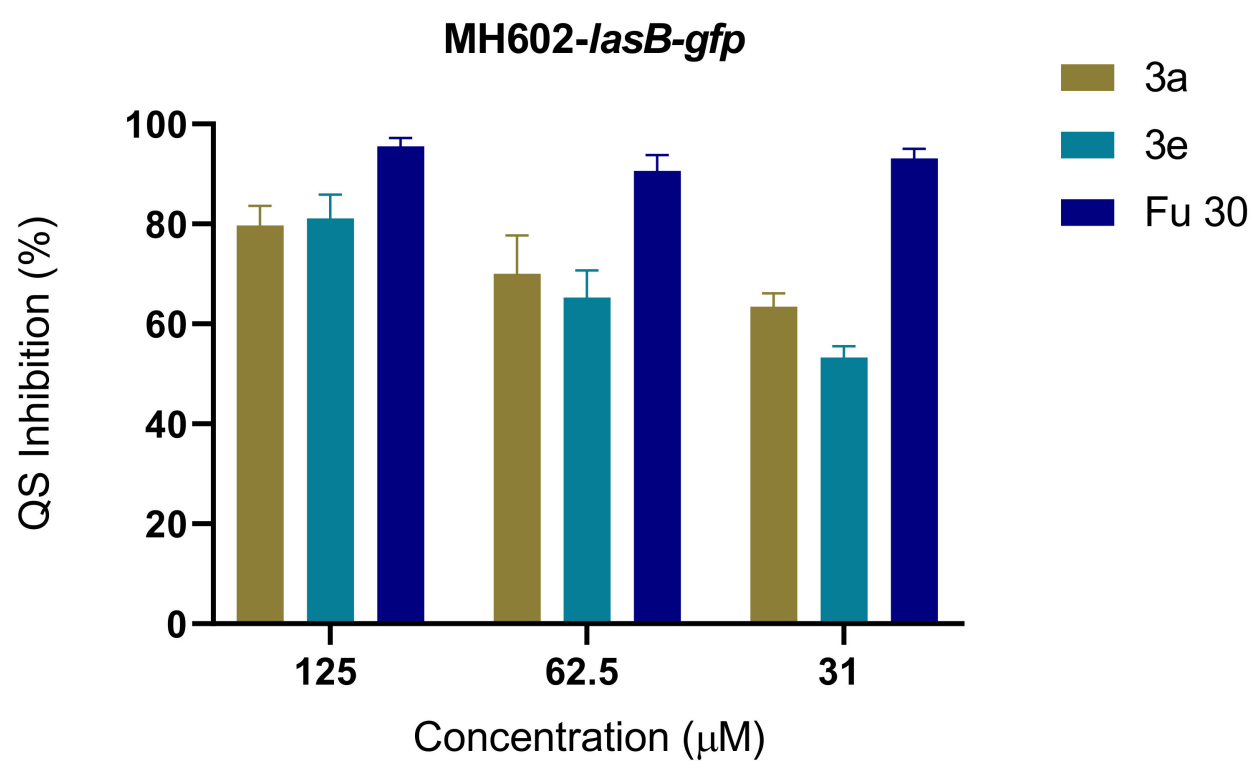

Figure 2. Comparison of \% LasR QS inhibition at different concentrations of inhibitors 3a, 3e, and reference Fu 30.

On the other hand, sulphur-containing compounds produced lower QS inhibition. The compound 4a (C-4 phenyl) showed $50 \%$ and $37 \%$ QS inhibition at $125 \mu \mathrm{M}$ and $31 \mu \mathrm{M}$, respectively. However, in this series, the most potent analogue was $4 \mathrm{~d}$ (4-Br phenyl) which exhibited QS inhibition of $74 \%$ at $125 \mu \mathrm{M}$ and $41 \%$ at $31 \mu \mathrm{M}$.

\subsection{Antibacterial Activity}

As these new compounds contain cationic selenourea and thiourea functionalities, we were interested to see if these compounds had antibacterial activity. When the com- 
pounds were screened for their minimum inhibitory concentrations (MICs), using the Gram-negative bacteria E. coli (Table 3), or their effect on the growth of $P$. aeruginosa (Supplementary Materials, File S48), none were able to reduce the growth by $90 \%$, the minimum growth inhibition to obtain a MIC. Selenium-containing compounds produced a high level (23-50\%) of growth inhibition of $P$. aeruginosa at $125 \mu \mathrm{M}$, while at $31 \mu \mathrm{M}$, the growth inhibition induced by the selenium-containing compounds was $<20 \%$, except for compound 3c, which still gave a growth inhibition of $23 \%$. This suggests that these selenium-containing compounds (except 3c) work predominantly as QS inhibitors at low concentrations.

Table 3. MIC determined for compounds against Gram-positive S. aureus (SA38) and Gram-negative bacteria (E. coli K12). NT-Not tested.

\begin{tabular}{cccc}
\hline \multirow{2}{*}{ Entry } & Compounds & \multicolumn{2}{c}{ MIC/MBC $(\mu \mathrm{M})$} \\
\cline { 2 - 4 } & & S. aureus $\mathbf{3 8}$ & E. coli K12 \\
\hline 1 & $3 \mathrm{a}$ & 31.2 & $>250$ \\
2 & $3 \mathrm{~b}$ & 125 & $\mathrm{NT}$ \\
3 & $3 \mathrm{c}$ & $\mathbf{1 5 . 6 / 3 1 . 2}$ & $>250$ \\
4 & $3 \mathrm{~d}$ & $>250$ & $\mathrm{NT}$ \\
5 & $3 \mathrm{e}$ & $\mathbf{1 5 . 6 / 6 2 . 4}$ & $>250$ \\
6 & $3 \mathrm{f}$ & 62.5 & $>250$ \\
7 & $3 \mathrm{~g}$ & 62.5 & $>250$ \\
8 & $3 \mathrm{~h}$ & $>250$ & $\mathrm{NT}$ \\
9 & $4 \mathrm{a}$ & $>250$ & $\mathrm{NT}$ \\
10 & $4 \mathrm{~b}$ & $>250$ & $\mathrm{NT}$ \\
11 & $4 \mathrm{c}$ & $>250$ & $\mathrm{NT}$ \\
12 & $4 \mathrm{~d}$ & $>250$ & $\mathrm{NT}$ \\
13 & $4 \mathrm{e}$ & $>250$ & $\mathrm{NT}$ \\
14 & $4 \mathrm{~g}$ & $>250$ & $\mathrm{NT}$ \\
15 & $4 \mathrm{f}$ & $>250$ & $\mathrm{NT}$ \\
16 & Gentamicin & $<0.4$ & $\mathrm{NT}$ \\
\hline
\end{tabular}

Nevertheless, when the compounds were screened for their minimum inhibitory concentration (MIC) against the Gram-positive S. aureus SA38 (isolated from a human corneal ulcer) (Table 3), the selenium-containing compounds ( $3 a-h)$ were able to reduce the growth of $S$. aureus. Compounds $3 \mathrm{c}$ and $3 \mathrm{e}$, which had $4-\mathrm{Cl}$ and $2-\mathrm{Cl}$ substitution, respectively, were the most potent with MICs of $15.6 \mu \mathrm{M}$. These did not show haemolysis at $100 \mu \mathrm{M}$. The corresponding $4-\mathrm{F}$ and $4-\mathrm{Br}$ derivatives $\mathbf{3 b}$ and $\mathbf{3 d}$ were less active, with MICs of $125 \mu \mathrm{M}$ and $>250 \mu \mathrm{M}$, respectively. The substitution of a methyl group at the 3-position of the lactam also led to significant loss of antimicrobial activity as compound $\mathbf{3 f}$ had a MIC of $62.5 \mu \mathrm{M}$ compared to MIC of $15.6 \mu \mathrm{M}$ for 3c. On the other hand, none of the corresponding sulphur analogues showed any activity at the maximum concentration of $250 \mu \mathrm{M}$. These results further confirm that selenium is critical for antibacterial activity.

\section{Conclusions}

In summary, a new series of selenourea and thiourea dihydropyrrol-2-one compounds were synthesised and screened for LasR QSI activity using the P. aeruginosa MH602-P $\mathrm{P}_{\text {las }} \mathrm{B}-\mathrm{gff} p$ reporter strain. The results showed that most of the compounds produced a concentrationdependent inhibition of las QS. Interestingly, selenium-containing compounds were significantly more effective inhibitors compared to the corresponding sulphur-containing analogues, most likely due to the presence of both selenium (and possibly also the cationic $+\mathrm{NH}_{2}$ ). Except for compounds $\mathbf{3} \mathbf{d}$ and $\mathbf{3 h}$, all the selenium analogues reduced the growth of S. aureus SA38, with derivatives $3 \mathrm{c}$ and 3e showing potent activity with MICs of $15.6 \mu \mathrm{M}$. The results indicated that the presence of selenium is essential for both QSI against Gramnegative P. aeruginosa and antibacterial activity against Gram-positive S. aureus. To the best of our knowledge, this is the first description of dual-acting organoselenium compounds 
as quorum-sensing inhibitors in Gram-negative (P. aeruginosa) and bactericidal activity in Gram-positive bacteria ( $S$. aureus). In the future, our efforts will focus on developing more potent selenium-based antibacterial compounds by structure-activity relationships, and we will also evaluate the effect of these compounds on the formation of bacterial biofilm and other virulence factors.

\section{Materials and Methods}

All the reagents and solvents were purchased from commercial sources (Combi-Blocks, Sigma-Aldrich, and Oakwood chemicals) and used without further purification. Reactions were performed using oven-dried glassware. Room temperature (rt) refers to the ambient temperature $\left(25^{\circ} \mathrm{C}\right)$. Progress of reactions was monitored by thin-layer chromatography (TLC) using precoated Merck silica gel 60 F254 plates and visualised using UV light $(254 \mathrm{~nm})$. Melting points of the new compounds were determined by an SRS MPA100 OptiMelt instrument and are reported without correction. IR spectra were recorded using a Cary 630 ATR FTIR spectrophotometer. High-resolution mass spectrometry (HRMS) was performed by electrospray (ESI) ionisation using a Thermo LTQ Orbitrap XL instrument at Bioanalytical Mass Spectrometry Facility (BMSF) of Mark Wainwright Analytical Centre, UNSW Sydney. ${ }^{1} \mathrm{H}$ and ${ }^{13} \mathrm{C}$-NMR were recorded in deuterated DMSO-d6) using Bruker Avance III 300 and Bruker Avance III $400 \mathrm{MHz}$ instruments (Bruker Pty Ltd, Preston, Australia) at $24{ }^{\circ} \mathrm{C}$. Chemical shifts $(\delta)$ are reported as relative to the corresponding solvent peak, with tetramethylsilane as the internal standard and quoted in parts per million (ppm). The coupling constants $(J)$ are reported in hertz $(\mathrm{Hz})$.

\subsection{General Procedure for Synthesis of Selenourea and Thiourea DHP Compounds}

The corresponding DHP bromide $(0.2 \mathrm{mmol})$ was added to a stirring mixture of selenourea or thiourea $(0.22 \mathrm{mmol})$ in acetone $(5 \mathrm{~mL})$ at $\mathrm{rt}$. The mixture was then heated to $60{ }^{\circ} \mathrm{C}$ for $1-6 \mathrm{~h}$. Progress of the reaction was monitored by TLC, and after completion of the reaction, the solid precipitate was collected by filtration or by evaporation under vacuum and was further purified by washing with diethyl ether $(10 \mathrm{~mL})$.

(Z)-(5-oxo-3-phenyl-1,5-dihydro-2H-pyrrol-2-ylidene) methyl carbamimidoselenoate hydrobromide (3a): Following the general procedure $\mathrm{A}$, the title product was obtained as a yellow solid (62 mg, 83\% yield); mp 146-149 ${ }^{\circ} \mathrm{C} ;{ }^{1} \mathrm{H}$ NMR (400 MHz, DMSO-d6) $\delta 10.51$ (s, 1H), 9.24 $(\mathrm{s}, 4 \mathrm{H}), 7.81-7.55(\mathrm{~m}, 2 \mathrm{H}), 7.55-7.24(\mathrm{~m}, 3 \mathrm{H}), 6.47(\mathrm{~d}, J=1.6 \mathrm{~Hz}, 1 \mathrm{H}), 6.28(\mathrm{~s}, 1 \mathrm{H}) .{ }^{13} \mathrm{C} \mathrm{NMR}$ (101 MHz, DMSO-d6) $\delta 170.40,165.78,150.14,145.72,131.06,129.68,128.86,122.46,94.19$. IR (ATR): $v_{\max }$ 3155-2985, 1710, 1638; ESI-HRMS $m / z$ : calcd for $\mathrm{C}_{12} \mathrm{H}_{11} \mathrm{~N}_{3} \mathrm{OSe}[\mathrm{M}+\mathrm{H}]^{+}$: 294.0140; found 294.0134.

(Z)-(3-(4-fluorophenyl)-5-oxo-1,5-dihydro-2H-pyrrol-2-ylidene)methyl carbamimidoselenoate hydrobromide ( $3 \mathbf{b})$ : The title product was obtained as a yellow solid (64 $\mathrm{mg}, 82 \%$ yield); $\mathrm{mp}$ 162-165 ${ }^{\circ} \mathrm{C} ;{ }^{1} \mathrm{H}$ NMR (400 MHz, DMSO-d6) $\delta 10.52$ (d, $\left.J=1.8 \mathrm{~Hz}, 1 \mathrm{H}\right), 9.25$ (s, 5H), 7.65 (dd, $J=8.7,5.5 \mathrm{~Hz}, 2 \mathrm{H}), 7.34(\mathrm{t}, J=8.9 \mathrm{~Hz}, 2 \mathrm{H}), 6.47(\mathrm{~d}, J=1.5 \mathrm{~Hz}, 1 \mathrm{H}), 6.25(\mathrm{~s}, 1 \mathrm{H}) .{ }^{13} \mathrm{C} \mathrm{NMR}$ (101 MHz, DMSO-d6) $\delta$ 170.32, 165.81, 164.18, 161.73, 149.04, 145.81, 131.25, 131.16, 127.52, $127.49,122.61,115.95,115.74,94.11$. IR (ATR): $v_{\max } 3243,3100,1681,1643 ;$ ESI-HRMS $m / z$ : calcd for $\mathrm{C}_{12} \mathrm{H}_{11} \mathrm{FN}_{3} \mathrm{OSe}[\mathrm{M}+\mathrm{H}]^{+}$: 312.0046; found 312.0042.

(Z)-(3-(4-chlorophenyl)-5-oxo-1,5-dihydro-2H-pyrrol-2-ylidene)methyl carbamimidoselenoate hydrobromide (3c): The title product was obtained as a yellow solid (71 $\mathrm{mg}, 85 \%$ yield); $\mathrm{mp}$ 170-175 ${ }^{\circ} \mathrm{C} ;{ }^{1} \mathrm{H}$ NMR (400 MHz, DMSO-d6) $\delta 10.56$ (s, 1H), 9.23 (s, 5H), 7.68-7.54 (m, 4H), $6.52(\mathrm{~d}, J=1.6 \mathrm{~Hz}, 1 \mathrm{H}), 6.28(\mathrm{~s}, 1 \mathrm{H}) .{ }^{13} \mathrm{C}$ NMR (101 MHz, DMSO-d6) $\delta 170.24,165.73,148.83$, 145.50, 134.54, 130.72, 129.88, 128.87, 122.95, 94.27. IR (ATR): $v_{\max }$ 3170-2993, 1684, 1640; ESI-HRMS $m / z$ : calcd for $\mathrm{C}_{12} \mathrm{H}_{11} \mathrm{ClN}_{3} \mathrm{OSe}[\mathrm{M}+\mathrm{H}]^{+}:$327.9750; found 327.9743 .

(Z)-(3-(4-bromophenyl)-5-oxo-1,5-dihydro-2H-pyrrol-2-ylidene)methyl carbamimidoselenoate hydrobromide (3d): The title product was obtained as a yellow solid (66 $\mathrm{mg}, 70 \%$ yield); $\mathrm{mp}$ 173-175 ${ }^{\circ} \mathrm{C} ;{ }^{1} \mathrm{H}$ NMR (400 MHz, DMSO-d6) $\delta 10.56$ (s, 1H), 9.23 (s, 4H), 7.71 (d, J = 8.5 Hz, $2 \mathrm{H}), 7.55(\mathrm{~d}, J=8.5 \mathrm{~Hz}, 2 \mathrm{H}), 6.53(\mathrm{~d}, J=1.6 \mathrm{~Hz}, 1 \mathrm{H}), 6.29(\mathrm{~s}, 1 \mathrm{H}) .{ }^{13} \mathrm{C} \mathrm{NMR}(101 \mathrm{MHz}$, DMSO-d6) $\delta$ 170.19, 165.68, 148.86, 145.35, 131.76, 130.92, 130.21, 123.24, 122.89, 94.27. IR 
(ATR): $v_{\max } 3165-2986,1702,1649$; ESI-HRMS $m / z$ : calcd for $\mathrm{C}_{12} \mathrm{H}_{11} \mathrm{BrN}_{3} \mathrm{OSe}[\mathrm{M}+\mathrm{H}]^{+}$: 371.9245; found 371.9237.

(Z)-(3-(2-chlorophenyl)-5-oxo-1,5-dihydro-2H-pyrrol-2-ylidene)methyl carbamimidoselenoate hydrobromide (3e): The title product was obtained as a yellow solid ( $69 \mathrm{mg}, 84 \%$ yield); $\mathrm{mp}$ $165-168{ }^{\circ} \mathrm{C} ;{ }^{1} \mathrm{H}$ NMR $(400 \mathrm{MHz}, \mathrm{DMSO}-d 6) \delta 10.65(\mathrm{~s}, 1 \mathrm{H}), 9.23(\mathrm{~s}, 4 \mathrm{H}), 7.63(\mathrm{dd}, J=7.8$, $1.4 \mathrm{~Hz}, 1 \mathrm{H}), 7.60-7.41(\mathrm{~m}, 3 \mathrm{H}), 6.49(\mathrm{~d}, J=1.5 \mathrm{~Hz}, 1 \mathrm{H}), 5.99(\mathrm{~s}, 1 \mathrm{H}) .{ }^{13} \mathrm{C}$ NMR $(101 \mathrm{MHz}$, DMSO-d6) $\delta 170.73,165.84,147.30,146.02,132.69,132.10,131.41,130.43,130.09,127.63$, 125.74, 94.75. ${ }^{77}$ Se NMR (76 MHz, DMSO-d6) $\delta 388.56$. IR (ATR): $v_{\max } 3156-3031,1689$, 1645; ESI-HRMS m/z: calcd for $\mathrm{C}_{12} \mathrm{H}_{10} \mathrm{ClN}_{3} \mathrm{OSe}[\mathrm{M}+\mathrm{H}]^{+}$: 327.9750; found 327.9750.

(Z)-(3-(4-chlorophenyl)-4-methyl-5-oxo-1,5-dihydro-2H-pyrrol-2-ylidene)methyl carbamimidoselenoate hydrobromide (3f): The title product was obtained as a light yellow solid (54 $\mathrm{mg}$, $63 \%$ yield); mp 180-182 ${ }^{\circ} \mathrm{C} ;{ }^{1} \mathrm{H}$ NMR (400 MHz, DMSO-d6) $\delta 10.58(\mathrm{~s}, 1 \mathrm{H}), 9.12(\mathrm{~d}, J=23.4$ $\mathrm{Hz}, 4 \mathrm{H}), 7.58(\mathrm{~d}, J=8.5 \mathrm{~Hz}, 2 \mathrm{H}), 7.50(\mathrm{~d}, J=8.5 \mathrm{~Hz}, 2 \mathrm{H}), 5.92(\mathrm{~s}, 1 \mathrm{H}), 1.85(\mathrm{~s}, 3 \mathrm{H}) .{ }^{13} \mathrm{C} \mathrm{NMR}$ (101 MHz, DMSO-d6) $\delta 171.51,166.66,147.65,142.19,134.20,131.88,131.82,129.89,129.18$, 91.15, 9.64. IR (ATR): $v_{\max } 3258-2976,1698,1640$; ESI-HRMS $m / z$ : calcd for $\mathrm{C}_{13} \mathrm{H}_{12} \mathrm{FNOS}$ $[\mathrm{M}+\mathrm{H}]^{+}: 341.9907$; found 341.9905 .

(Z)-(3-(2-fluorophenyl)-5-oxo-1,5-dihydro-2H-pyrrol-2-ylidene)methyl carbamimidoselenoate hydrobromide (3g): The title product was obtained as a yellow solid (64 $\mathrm{mg}, 82 \%$ yield); $\mathrm{mp}$ $159-161{ }^{\circ} \mathrm{C} ;{ }^{1} \mathrm{H}$ NMR $(300 \mathrm{MHz}$, DMSO-d6) $\delta 10.63(\mathrm{~s}, 1 \mathrm{H}), 9.23(\mathrm{~s}, 4 \mathrm{H}), 7.62-7.51(\mathrm{~m}, 2 \mathrm{H})$, 7.38-7.30 (m, 2H), $6.50(\mathrm{~s}, 1 \mathrm{H}), 6.17(\mathrm{~s}, 1 \mathrm{H}) .{ }^{13} \mathrm{C}$ NMR (76 MHz, DMSO-d6) $\delta 170.28,165.55$, $160.45,154.88,145.32,143.41,131.78,131.66,131.47,125.13,124.69,118.69,116.40,116.11$, 94.39. IR (ATR): $v_{\max } 3162-3013,1690,1647$; ESI-HRMS $m / z$ : calcd for $\mathrm{C}_{12} \mathrm{H}_{11} \mathrm{FN}_{3} \mathrm{OSe}$ $[\mathrm{M}+\mathrm{H}]^{+}:$: 312.0046; found 312.0040.

(Z)-(3-(4-methoxyphenyl)-5-oxo-1,5-dihydro-2H-pyrrol-2-ylidene)methyl carbamimidoselenoate hydrobromide ( $3 \mathbf{h}$ ): The title product was obtained as a yellow solid ( $65 \mathrm{mg}, 80 \%$ yield); $\mathrm{mp}$ $138-140{ }^{\circ} \mathrm{C} ;{ }^{1} \mathrm{H}$ NMR $(600 \mathrm{MHz}$, DMSO-d6) $\delta 10.41(\mathrm{~s}, 1 \mathrm{H}), 9.22(\mathrm{~s}, 4 \mathrm{H}), 7.55(\mathrm{~d}, J=8.6 \mathrm{~Hz}$, $2 \mathrm{H}), 7.08-7.02(\mathrm{~m}, 2 \mathrm{H}), 6.38(\mathrm{~d}, J=1.6 \mathrm{~Hz}, 1 \mathrm{H}), 6.26(\mathrm{~s}, 1 \mathrm{H}), 3.81(\mathrm{~s}, 3 \mathrm{H}) .{ }^{13} \mathrm{C}$ NMR $(151 \mathrm{MHz}$, DMSO-d6) $\delta$ 170.54, 165.83, 160.47, 149.88, 146.03, 130.33, 123.35, 121.10, 114.31, 93.67, 55.38. IR (ATR): $v_{\max } 3102-2992,1681,1648$; ESI-HRMS $m / z$ : calcd for $\mathrm{C}_{13} \mathrm{H}_{13} \mathrm{~N}_{3} \mathrm{O}_{2} \mathrm{Se}[\mathrm{M}+\mathrm{H}]^{+}$: 324.0246; found 324.0241.

(Z)-(5-oxo-3-phenyl-1,5-dihydro-2H-pyrrol-2-ylidene)methyl carbamimidothioate hyrobromide (4a): The title product was obtained as a yellow solid (45 $\mathrm{mg}, 69 \%$ yield); $\mathrm{mp}$ 172-175 ${ }^{\circ} \mathrm{C} ;{ }^{1} \mathrm{H}$ NMR (400 MHz, DMSO-d6) $\delta 10.75(\mathrm{~s}, 1 \mathrm{H}), 9.14(\mathrm{~d}, J=23.6 \mathrm{~Hz}, 4 \mathrm{H})$, 7.65-7.56 (m, 2H), 7.55-7.46 (m, 3H), $6.51(\mathrm{~d}, J=1.5 \mathrm{~Hz}, 1 \mathrm{H}), 5.96(\mathrm{~s}, 1 \mathrm{H}) .{ }^{13} \mathrm{C}$ NMR (101 MHz, DMSO-d6) \& 170.33, 167.87, 150.28, 147.24, 130.87, 129.75, 128.86, 122.96, 93.01. IR (ATR): $v_{\max } 3251-3055,1684,1650$; ESI-HRMS $m / z$ : calcd for $\mathrm{C}_{12} \mathrm{H}_{11} \mathrm{~N}_{3} \mathrm{OS}[\mathrm{M}+\mathrm{H}]^{+}$: 246.0696; found 246.0693 .

(Z)-(3-(4-fluorophenyl)-5-oxo-1,5-dihydro-2H-pyrrol-2-ylidene)methyl carbamimidothioate hyrobromide (4b) The title product was obtained as a yellow solid $(65 \mathrm{mg}, 94 \%$ yield); mp 175-177 ${ }^{\circ} \mathrm{C} ;{ }^{1} \mathrm{H}$ NMR (600 MHz, DMSO-d6) $\delta 10.74(\mathrm{~s}, 1 \mathrm{H}), 9.17(\mathrm{~d}, J=41.0 \mathrm{~Hz}, 4 \mathrm{H})$, 7.69-7.63 (m, 2H), 7.37-7.32 (m, 2H), $6.50(\mathrm{~d}, J=1.6 \mathrm{~Hz}, 1 \mathrm{H}), 5.93(\mathrm{~s}, 1 \mathrm{H}) .{ }^{13} \mathrm{C}$ NMR (151 MHz, DMSO-d6) $\delta 170.70,168.32,164.26,162.62,149.65,147.77,131.70,131.65,127.76$, 127.74, 123.53, 116.38, 116.23, 93.40. IR (ATR): $v_{\max }$ 3251-3054, 1690, 1650; ESI-HRMS $m / z$ : calcd for $\mathrm{C}_{12} \mathrm{H}_{10} \mathrm{FN}_{3} \mathrm{OS}[\mathrm{M}+\mathrm{H}]^{+}: 264.0601$; found 264.0598 .

(Z)-(3-(4-chlorophenyl)-5-oxo-1,5-dihydro-2H-pyrrol-2-ylidene)methyl mcarbamimidothioate hydrobromide (4c): The title product was obtained as a light orange solid $(52 \mathrm{mg}, 71 \%$ yield); mp 149-151 ${ }^{\circ} \mathrm{C} ;{ }^{1} \mathrm{H}$ NMR (400 MHz, DMSO-d6) $\delta 10.78(\mathrm{~s}, 1 \mathrm{H}), 9.12$ (d, J = 21.5 Hz, $4 \mathrm{H}), 7.67-7.60(\mathrm{~m}, 2 \mathrm{H}), 7.60-7.54(\mathrm{~m}, 2 \mathrm{H}), 6.54(\mathrm{~d}, J=1.5 \mathrm{~Hz}, 1 \mathrm{H}), 5.95(\mathrm{~s}, 1 \mathrm{H}) ;{ }^{13} \mathrm{C} \mathrm{NMR}$ (101 MHz, DMSO-d6) $\delta 170.16,167.77,148.99,146.98,134.62,130.72,129.69,128.88,123.44$, 93.12. IR (ATR): $v_{\max } 3155-2991,1685,1643$; ESI-HRMS $m / z$ : calcd for $\mathrm{C}_{12} \mathrm{H}_{11} \mathrm{ClN}_{3} \mathrm{OS}$ $[\mathrm{M}+\mathrm{H}]^{+}:$: 280.0306; found 280.0306 .

(Z)-(3-(4-bromophenyl)-5-oxo-1,5-dihydro-2H-pyrrol-2-ylidene)methyl carbamimidothioate hydrobromide (4d): The title product was obtained as a light orange solid $(78 \mathrm{mg}, 96 \%$ yield); mp 169-171 ${ }^{\circ} \mathrm{C} ;{ }^{1} \mathrm{H}$ NMR (300 MHz, DMSO-d6) $\delta 10.78$ (s, 1H), 9.13 (s, 4H), 7.72 
$(\mathrm{d}, J=8.5 \mathrm{~Hz}, 2 \mathrm{H}), 7.56(\mathrm{~d}, J=8.5 \mathrm{~Hz}, 2 \mathrm{H}), 6.56(\mathrm{~d}, J=1.5 \mathrm{~Hz}, 1 \mathrm{H}), 5.96(\mathrm{~s}, 1 \mathrm{H}) .{ }^{13} \mathrm{C} \mathrm{NMR}$ (76 MHz, DMSO-d6) $\delta$ 170.12, 167.72, 149.03, 146.86, 131.77, 130.92, 130.02, 123.39, 123.33, 93.10. IR (ATR): $v_{\max } 3140-2959,1696,1651$; ESI-HRMS $m / z$ : calcd for $\mathrm{C}_{12} \mathrm{H}_{11} \mathrm{BrN}_{3} \mathrm{OS}$ $[\mathrm{M}+\mathrm{H}]^{+}$: 323.9801; found 323.9802.

(Z)-(3-(2-fluorophenyl)-5-oxo-1,5-dihydro-2H-pyrrol-2-ylidene)methyl carbamimidothioate hydrobromide (4e): The title product was obtained as a yellow solid (45 $\mathrm{mg}, 66 \%$ yield); mp 166-168 ${ }^{\circ} \mathrm{C} ;{ }^{1} \mathrm{H}$ NMR (400 MHz, DMSO-d6) $\delta 10.85$ (s, 1H), 9.14 (s, 4H), $7.65-7.50$ $(\mathrm{m}, 2 \mathrm{H}), 7.43-7.29(\mathrm{~m}, 2 \mathrm{H}), 6.53(\mathrm{~s}, 1 \mathrm{H}), 5.84(\mathrm{~s}, 1 \mathrm{H}) .{ }^{13} \mathrm{C}$ NMR (101 MHz, DMSO-d6) $\delta$ $183.86,170.21,167.66,160.47,158.00,146.79,143.50,131.83,131.74,131.44,125.63,125.60$, $124.69,124.66,118.48,118.34,116.37,116.15,93.22$. IR (ATR): $v_{\max } 3159-3028,1691,1650$; ESI-HRMS $m / z$ : calcd for $\mathrm{C}_{12} \mathrm{H}_{10} \mathrm{FN}_{3} \mathrm{OS}[\mathrm{M}+\mathrm{H}]^{+}: 264.0601$; found 264.0600 .

(Z)-(3-(2-chlorophenyl)-5-oxo-1,5-dihydro-2H-pyrrol-2-ylidene)methyl carbamimidothioate hydrobromide (4f): The title product was obtained as a yellow solid (35 $\mathrm{mg}, 48 \%$ yield); mp 187-190 ${ }^{\circ} \mathrm{C} ;{ }^{1} \mathrm{H}$ NMR (300 MHz, DMSO-d6) $\delta 10.87$ (s, 1H), 9.12 (s, 4H), $7.67-7.41$ (m, 4H), 6.52 (s, 1H), 5.65 (s, 1H). ${ }^{13} \mathrm{C}$ NMR (76 MHz, DMSO-d6) $\delta 170.19,167.52,146.98,146.91$, $132.23,131.64,130.99,129.97,129.38,127.17,125.83,93.09$. IR (ATR): $v_{\max } 3225-3040,1690$, 1651; ESI-HRMS m/z: calcd for $\mathrm{C}_{12} \mathrm{H}_{11} \mathrm{ClN}_{3} \mathrm{OS}[\mathrm{M}+\mathrm{H}]^{+}: 280.0306$; found 280.0303 .

(Z)-(5-oxo-3-(3-(trifluoromethyl)phenyl)-1,5-dihydro-2H-pyrrol-2-ylidene)methyl carbamimidothioate hydrobromide (4g): The title product was obtained as a yellow solid $(60 \mathrm{mg}, 76 \%$ yield); mp 168-170 ${ }^{\circ} \mathrm{C} ;{ }^{1} \mathrm{H}$ NMR (400 MHz, DMSO-d6) $\delta 10.84$ (s, 1H), 9.13 (d, J = 22.8 Hz, $4 \mathrm{H}), 7.95(\mathrm{~s}, 1 \mathrm{H}), 7.94-7.85(\mathrm{~m}, 2 \mathrm{H}), 7.76(\mathrm{t}, J=7.8 \mathrm{~Hz}, 2 \mathrm{H}), 6.67(\mathrm{~d}, J=1.5 \mathrm{~Hz}, 1 \mathrm{H}), 5.94(\mathrm{~s}$, 1H). ${ }^{13} \mathrm{C}$ NMR $(151 \mathrm{MHz}$, DMSO-d6) $\delta$ 170.04, 167.78, 148.63, 146.93, 132.97, 131.87, 129.96, $129.72,129.51,126.24,125.48,125.45,124.85,124.42,123.04,93.27$. IR (ATR): $v_{\max } 3120-3008$, 1710, 1641; ESI-HRMS $m / z$ : calcd for $\mathrm{C}_{13} \mathrm{H}_{10} \mathrm{FN}_{3} \mathrm{OS}[\mathrm{M}+\mathrm{H}]^{+}$: 314.0569 ; found 314.0565 .

\subsection{Quorum-Sensing Inhibition Assay}

P. aeruginosa $\mathrm{MH602} \mathrm{P}_{\text {las }} \mathrm{B}:: g f p(\mathrm{ASV})$ reporter strain, which harbours a chromosomal fusion of the $l a s B$ promoter to an unstable $g f p$ gene and responds to the AHL 3-oxododecanoyl homoserine lactone (3oxo-C12-HSL), was used to evaluate the QSI activity of the synthesised compounds on QS signalling. An overnight culture was prepared in Luria-Bertani (LB10) media supplemented with gentamycin $(40 \mu \mathrm{M})$. This culture was then diluted (1:100) in TSB/LB10 (4:1) medium supplemented with gentamycin $(40 \mu \mathrm{M})$, and $200 \mu \mathrm{L}$ of aliquots were dispensed to flat-bottom 96-well plate wells (Costar). The culture was supplemented with varying concentrations of test compounds dissolved in dimethyl sulfoxide, with the final concentrations of test compounds of 125,63 , and $31 \mu \mathrm{M}$. Wells with bacterial culture but no compound were used as a negative control, while wells supplemented with the furanone Fu-30 were used as a positive control. Plates were incubated for $15 \mathrm{~h}$ at $37{ }^{\circ} \mathrm{C}$ with shaking at $150 \mathrm{rpm}$. After incubation, the reading of the fluorescence of GFP (excitation, $485 \mathrm{~nm}$; emission, $520 \mathrm{~nm}$ ) and cell growth (OD at $600 \mathrm{~nm}$ ) was taken in a plate reader (FLUOstar Omega, BMG Labtech, Ortenberg, Germany).

\subsection{Analysis of the Minimum Inhibitory and Minimum Bactericidal Concentration of Compounds}

The antimicrobial activity of compounds was evaluated using the broth microdilution assay described by The Clinical and Laboratory Standards Institute. Briefly, Staphylococcus aureus SA38 or Escherichia coli $\mathrm{K} 12$ were grown to mid-log phase (18-24 h incubation) in Muller-Hinton broth (MHB) with shaking at $120 \mathrm{rpm}$ at $37^{\circ} \mathrm{C}$. Following incubation, bacteria were washed two times using phosphate-buffered saline ( $\mathrm{pH} 7.4)$ with centrifugation for $10 \mathrm{~min}$ after each wash. After washing, bacteria were diluted with fresh MHB to a turbidity of OD600 of 0.1 (which gave $1 \times 10^{8} \mathrm{cfu} / \mathrm{mL}$ on retrospective plate counts) and then further diluted to achieve $1 \times 10^{6} \mathrm{cfu} / \mathrm{mL}$ as a final bacterial concentration. Each compound was added at concentrations ranging from 250 to $3.9 \mu \mathrm{M}$ through serial twofold dilution. Wells containing only bacteria and without any compound were used as a negative control and only MHB media as blank. Gentamicin was used as a positive control. The microtiter plate was wrapped with paraffin to prevent evaporation and incubated with 
shaking at $120 \mathrm{rpm}$ and $37^{\circ} \mathrm{C}$ for $18-24 \mathrm{~h}$. After incubation, spectrophotometric reading of the wells was taken at $600 \mathrm{~nm}$ using a FLUOstar Omega (BMG Labtech, Mornington, Victoria, Australia). The minimum inhibitory concentration (MIC) was defined as the lowest concentration of the compounds that produce $>90 \%$ inhibition of microbial growth Each experiment was performed with two independent repeats. The MBC was determined as the lowest concentration of compound that reduced bacterial growth by $>99.99 \%$ after the enumeration of viable bacteria by plate counts compared to bacteria grown without any compounds.

\subsection{Lysis of Horse Red Blood Cells}

The haemolytic activities of compounds $3 \mathbf{c}$ and $3 \mathbf{e}$ were determined using horse red blood cells (HRBCs; Sigma) as described previously [37]. The HRBCs were washed three times with PBS at $470 \times g$ for $5 \mathrm{~min}$. Compounds $(100 \mu \mathrm{M}, 50 \mu \mathrm{M}$ and $25 \mu \mathrm{M}$, in PBS) were added to the washed HRBCs and incubated at $37^{\circ} \mathrm{C}$ for $4 \mathrm{~h}$. After incubation, the cells were pelleted at $1057 \times g$ for $5 \mathrm{~min}$, and the supernatant was removed to assess the release of haemoglobin by measuring OD540nm. HRBCs in PBS and HRBCs in distilled water were used as negative (diluent) and positive controls to achieve $0 \%$ and $100 \%$ lysis, respectively. The relative OD of HRBCs treated with compounds $3 \mathbf{c}$ and $3 \mathbf{e}$ were compared to that treated with distilled water were used to determine the relative percentage of haemolysis. Two separate trials were carried out in triplicates.

$\%$ haemolysis $=($ absorbance of test compound $)-($ absorbance of diluent $)$ / (absorbance of positive control) - (absorbance of diluent $) * 100$.

Supplementary Materials: The following are available online at https:/ / www.mdpi.com/2079-6 382/10/3/321/s1, Figures S2-S32: ${ }^{1} \mathrm{H}$ and ${ }^{13} \mathrm{C}$ NMR spectra of the compounds; Figure S12: ${ }^{77} \mathrm{Se}$ NMR spectrum of compound 3e; Figures S33-S47: HRMS data of the compounds; Figures S48-S52: IR Spectra of compounds; Figure S53: Growth inhibition data (P. aeruginosa MH602).

Author Contributions: N.K., M.D.P.W., and D.S.B. planned and designed the experiments. T.T.Y., R.K., and T.D. provided the data from biology screening. S.S. performed the chemistry experiments, analysed, and interpreted the data and wrote the paper. B.A. and G.I. help in proofreading the manuscript. N.K. supported financially. All authors have read and agreed to the published version of the manuscript.

Funding: This work was supported by a Discovery Project from Australian Research Council grant (DP180100845).

Institutional Review Board Statement: Not applicable.

Informed Consent Statement: Not applicable.

Data Availability Statement: Data is contained within the article and Supplementary Materials.

Acknowledgments: We thank the NMR and BMSF facility, UNSW Sydney. The authors would like to acknowledge the University of Sydney, Australia. S. Sabir would like to acknowledge UNSW TFS scholarship.

Conflicts of Interest: The authors declare no conflict of interest.

\section{References}

1. Kadri, S.S. Key Takeaways From the U.S. CDC's 2019 Antibiotic Resistance Threats Report for Frontline Providers. Crit. Care Med. 2020, 48, 939-945. [CrossRef]

2. World Health Organization. Antimicrobial Resistance: Global Report on Surveillance; World Health Organization: Geneva, Switzerland, 2014.

3. Kohanski, M.A.; Dwyer, D.J.; Collins, J.J. How antibiotics kill bacteria: From targets to networks. Nat. Rev. Microbiol. 2010, 8, 423-435. [CrossRef] [PubMed]

4. Walsh, C. Antibiotics: Actions, Origins, Resistance; American Society for Microbiology (ASM): Washington, DC, USA, 2003.

5. Holmes, A.H.; Moore, L.S.P.; Sundsfjord, A.; Steinbakk, M.; Regmi, S.; Karkey, A.; Guerin, P.J.; Piddock, L.J.V. Understanding the mechanisms and drivers of antimicrobial resistance. Lancet 2016, 387, 176-187. [CrossRef] 
6. Livermore, D.M. Multiple Mechanisms of Antimicrobial Resistance in Pseudomonas aeruginosa: Our Worst Nightmare? Clin. Infect. Dis. 2002, 34, 634-640. [CrossRef]

7. Tenover, F.C. Mechanisms of Antimicrobial Resistance in Bacteria. Am. J. Med. 2006, 119, S3-S10. [CrossRef]

8. Blair, J.M.A.; Webber, M.A.; Baylay, A.J.; Ogbolu, D.O.; Piddock, L.J.V. Molecular mechanisms of antibiotic resistance. Nat. Rev. Microbiol. 2015, 13, 42-51. [CrossRef] [PubMed]

9. Årdal, C.; Balasegaram, M.; Laxminarayan, R.; McAdams, D.; Outterson, K.; Rex, J.H.; Sumpradit, N. Antibiotic development -Economic, regulatory and societal challenges. Nat. Rev. Microbiol. 2020, 18, 267-274. [CrossRef]

10. Papenfort, K.; Bassler, B.L. Quorum sensing signal-response systems in Gram-negative bacteria. Nat. Rev. Microbiol. 2016, 14, 576-588. [CrossRef]

11. Waters, C.M.; Bassler, B.L. QUORUM SENSING: Cell-to-Cell Communication in Bacteria. Annu. Rev. Cell Dev. Biol. 2005, 21, 319-346. [CrossRef] [PubMed]

12. Schuster, M.; Greenberg, E.P. A network of networks: Quorum-sensing gene regulation in Pseudomonas aeruginosa. Int. J. Med. Microbiol. 2006, 296, 73-81. [CrossRef]

13. Pesci, E.C.; Pearson, J.P.; Seed, P.C.; Iglewski, B.H. Regulation of las and rhl quorum sensing in Pseudomonas aeruginosa. J. Bacteriol. 1997, 179, 3127-3132. [CrossRef]

14. Gilbert, K.B.; Kim, T.H.; Gupta, R.; Greenberg, E.P.; Schuster, M. Global position analysis of the Pseudomonas aeruginosa quorumsensing transcription factor LasR. Mol. Microbiol. 2009, 73, 1072-1085. [CrossRef]

15. Heurlier, K.; Dénervaud, V.; Haenni, M.; Guy, L.; Krishnapillai, V.; Haas, D. Quorum-Sensing-Negative (lasR) Mutants of Pseudomonas aeruginosa Avoid Cell Lysis and Death. J. Bacteriol. 2005, 187, 4875-4883. [CrossRef]

16. Chang, Y.; Wang, P.-C.; Ma, H.-M.; Chen, S.-Y.; Fu, Y.-H.; Liu, Y.-Y.; Wang, X.; Yu, G.-C.; Huang, T.; Hibbs, D.E.; et al. Design, synthesis and evaluation of halogenated furanone derivatives as quorum sensing inhibitors in Pseudomonas aeruginosa. Eur. J. Pharm. Sci. 2019, 140, 105058. [CrossRef]

17. Ho, K.K.; Kutty, S.K.; Chan, D.; Chen, R.; Willcox, M.D.; Kumar, N. Development of fimbrolides, halogenated furanones and their derivatives as antimicrobial agents. In Antibacterial Surfaces; Springer: Heidelberg, Germany, 2015; pp. 149-170.

18. De Nys, R.; Givskov, M.; Kumar, N.; Kjelleberg, S.; Steinberg, P. Furanones. In Antifouling Compounds; Springer: New York, NY, USA, 2006; pp. 55-86.

19. Grandclément, C.; Tannières, M.; Moréra, S.; Dessaux, Y.; Faure, D.D. Quorum quenching: Role in nature and applied developments. FEMS Microbiol. Rev. 2016, 40, 86-116. [CrossRef] [PubMed]

20. Almohaywi, B.; Yu, T.T.; Iskander, G.; Chan, D.S.; Ho, K.K.; Rice, S.; Black, D.S.; Griffith, R.; Kumar, N. Dihydropyrrolones as bacterial quorum sensing inhibitors. Bioorg. Med. Chem. Lett. 2019, 29, 1054-1059. [CrossRef]

21. Sabir, S.; Suresh, D.; Subramoni, S.; Das, T.; Bhadbhade, M.; Black, D.S.; Rice, S.A.; Kumar, N. Thioether-linked dihydropyrrol2-one analogues as PqsR antagonists against antibiotic resistant Pseudomonas aeruginosa. Bioorg. Med. Chem. 2021, $31,115967$. [CrossRef] [PubMed]

22. Mugesh, G.; Du Mont, W.-W.; Sies, H. Chemistry of Biologically Important Synthetic Organoselenium Compounds. Chem. Rev. 2001, 101, 2125-2180. [CrossRef] [PubMed]

23. Reich, H.J.; Hondal, R.J. Why Nature Chose Selenium. ACS Chem. Biol. 2016, 11, 821-841. [CrossRef]

24. Jain, V.K.; Priyadarsini, K.I. Organoselenium Compounds in Biology and Medicine: Synthesis, Biological and Therapeutic Treatments; Royal Society of Chemistry: London, UK, 2017.

25. Mugesh, G.; Singh, H.B. Synthetic organoselenium compounds as antioxidants: Glutathione peroxidase activity. Chem. Soc. Rev. 2000, 29, 347-357. [CrossRef]

26. Alcolea, V.; Plano, D.; Karelia, D.N.; Palop, J.A.; Amin, S.; Sanmartín, C.; Sharma, A.K. Novel seleno-and thio-urea derivatives with potent in vitro activities against several cancer cell lines. Eur. J. Med. Chem. 2016, 113, 134-144. [CrossRef] [PubMed]

27. Bartolini, D.; Sancineto, L.; de Bem, A.F.; Tew, K.D.; Santi, C.; Radi, R.; Toquato, P.; Galli, F. Selenocompounds in cancer therapy: An overview. In Advances in Cancer Research; Elsevier: Amsterdam, The Netherlands, 2017; Volume 136, pp. $259-302$.

28. Sancineto, L.; Piccioni, M.; De Marco, S.; Pagiotti, R.; Nascimento, V.; Braga, A.L.; Santi, C.; Pietrella, D. Diphenyl diselenide derivatives inhibit microbial biofilm formation involved in wound infection. BMC Microbiol. 2016, 16, 220. [CrossRef] [PubMed]

29. Pietrella, D. Antimicrobial activity of organoselenium compounds. In Organoselenium Chemistry: Between Synthesis and Biochemistry; Bentham Books: Sharjah, United Arab Emirates, 2014; pp. 328-344.

30. Pietka-Ottlik, M.; Burda-Grabowska, M.; Woźna, M.; Waleńska, J.; Kaleta, R.; Zaczyńska, E.; Piasecki, E.; Giurg, M. Synthesis of new alkylated and methoxylated analogues of ebselen with antiviral and antimicrobial properties. ARKIVOC 2017, 2017, 546-556. [CrossRef]

31. Sharma, N.; Kumar, S.; Maurya, I.K.; Bhasin, K.K.; Verma, A.; Wangoo, N.; Bhasin, A.K.K.; Mehta, S.K.; Kumar, S.; Sharma, R.K. Synthesis, structural analysis, antimicrobial evaluation and synergistic studies of imidazo[1,2-a]pyrimidine chalcogenides. RSC Adv. 2016, 6, 114224-114234. [CrossRef]

32. Sancineto, L.; Mariotti, A.; Bagnoli, L.; Marini, F.; DeSantis, J.; Iraci, N.; Santi, C.; Pannecouque, C.; Tabarrini, O. Design and Synthesis of DiselenoBisBenzamides (DISeBAs) as Nucleocapsid Protein 7 (NCp7) Inhibitors with anti-HIV Activity. J. Med. Chem. 2015, 58, 9601-9614. [CrossRef] 
33. Sartori, G.; Jardim, N.S.; Marcondes Sari, M.H.; Dobrachinski, F.; Pesarico, A.P.; Rodrigues, L.C., Jr.; Cargnelutti, J.; Flores, E.F.; Prigol, M.; Nogueira, C.W. Antiviral action of diphenyl diselenide on herpes simplex virus 2 infection in female BALB/c mice. J. Cell. Biochem. 2016, 117, 1638-1648. [CrossRef] [PubMed]

34. Amosova, S.V.; Novokshonova, I.A.; Penzik, M.V.; Filippov, A.S.; Albanov, A.I.; Potapov, V.A. Reaction of 2-bromomethyl-1,3thiaselenole with thiourea: En route to the first representatives of 2-(organylsulfanyl)-2,3-dihydro-1,4-thiaselenines. Tetrahedron Lett. 2017, 58, 4381-4383. [CrossRef]

35. Hentzer, M.; Riedel, K.; Rasmussen, T.B.; Heydorn, A.; Andersen, J.B.; Parsek, M.R.; Rice, S.A.; Eberl, L.; Molin, S.; Høiby, N.; et al. Inhibition of quorum sensing in Pseudomonas aeruginosa biofilm bacteria by a halogenated furanone compound. Microbiology 2002, 148, 87-102. [CrossRef]

36. Manefield, M.; Rasmussen, T.B.; Henzter, M.; Andersen, J.B.; Steinberg, P.; Kjelleberg, S.; Givskov, M. Halogenated furanones inhibit quorum sensing through accelerated LuxR turnover. Microbiology 2002, 148, 1119-1127. [CrossRef]

37. Yasir, M.; Dutta, D.; Willcox, M.D.P. Mode of action of the antimicrobial peptide Mel4 is independent of Staphylococcus aureus cell membrane permeability. PLoS ONE 2019, 14, e0215703. [CrossRef] [PubMed] 